\section{Cahiers de Narratologie}

Analyse et théorie narratives

$10.1 \mid 2001$

La voix narrative

\title{
La voix en question : L'Innommable de Beckett
}

\author{
Suzanne Allaire
}

\section{OpenEdition}

\section{Journals}

Electronic version

URL: http://journals.openedition.org/narratologie/6915

DOI: 10.4000/narratologie.6915

ISSN: 1765-307X

\section{Publisher}

LIRCES

\section{Printed version}

Date of publication: 1 January 2001

Number of pages: 63-75

ISBN: 2914561032

ISSN: 0993-8516

\section{Electronic reference}

Suzanne Allaire, "La voix en question : L'Innommable de Beckett", Cahiers de Narratologie [Online], 10.1 । 2001, Online since 16 October 2014, connection on 23 February 2021. URL: http://

journals.openedition.org/narratologie/6915 ; DOI: https://doi.org/10.4000/narratologie.6915 


\title{
LA VOIX EN QUESTION : L'INNOMMABLE DE BECKETT
}

\author{
Suzanne ALLAIRE \\ Université Haute Bretagne, Rennes 2
}

Aborder le roman sous l'angle de la voix narrative, ce n'est pas seulement, pour cerner l'acte de raconter, centrer le regard sur « l'instance productrice du discours », c'est aussi, passant du discours en général à l'une de ses modalités, « le discours narratif »', identifier à partir des traces qu'en porte le récit la présence textuelle d'un narrateur, point d'origine de l'histoire racontée. Or l'on sait que les romans de Beckett, sans cesser de s'inscrire dans l'univers de la fiction, se caractérisent par la production d'un discours romanesque très subversif. Et la désagrégation du récit pose alors le problème crucial de l'identité, et de l'identification, de cette "instance productrice » dès lors que le discours qui lui donne statut d'existence n'est plus à proprement parler narratif. Telle est bien la difficulté que soulève la lecture de L'Innommable ${ }^{2}$ : ce texte où s'opère, patiente, systématique, la destruction des ressorts du récit, est aussi celui qui, par la promotion du mot « voix » et par son questionnement sémantique, témoigne de bout en bout de l'intérêt que porte Beckett à l'activité verbale, une activité qui déborde de toutes parts les frontières de la narration. Comment nommer alors « l'instance productrice du discours »? Instance énonciative ? Instance fondée sur les ruines de l'instance narratoriale ? Ou tout simplement «cette voix qui parle » comme le fait Beckett? On ne saurait éluder une question de cette importance car ce sont, avec les

${ }^{1}$ Sur ces notions, comme sur celle de choix vocal, voir Genette, Figures III, Seuil, 1972, p. 226 et Nouveau discours du récit, Seuil, 1983 , p. 52.

${ }^{2}$ L'Innommable, Minuit, 1953. Toutes les citations qui suivent sont tirées de ce livre. La page de référence est précisée entre parenthèses. 
termes d'énonciation et de narration, les rapports de la parole et du récit qui sont en cause. Et si on a pu avec justesse noter qu'il est difficile de séparer dans ce livre le versant fictionnel du champ critique qui lui sert de commentaire, et ce en l'absence de ce que Greimas appelait le "débrayage du discours », il n'en reste pas moins que le détenteur de la parole, assumant pleinement son rôle de maître du jeu verbal, ne saurait se confondre avec la figure d'un narrateur qu'il dénonce ou révoque.

Rappelons rapidement le « choix vocal » dont relève L'Innommable: texte à une seule voix, présenté comme un soliloque, qui, conduit au présent et dans l'indéfinition de ce temps - «Quand maintenant ? » (7) - se donne à lire dans la contemporanéité même de son déroulement : "discours immédiat » donc (Genette 1983), ou " monologue autonome » (Cohn 1981)3. Assumé de plus et d'emblée par le pronom je, et délimité par une série d'exclusions, - «j'ai l'air de parler, ce n'est pas moi, de moi, ce n'est pas de moi » (7) -, c'est un roman qui s'enferme dans la parole de quelqu'un, et ce quelqu'un se parle en parlant de soi : «Moi que voici, moi qui suis ici, qui ne peux pas parler, ne peux pas penser et qui dois parler, donc penser peut-être un peu, ne le peux seulement par rapport à moi qui suis ici, à ici où je suis, mais le peux un peu, suffisamment, je ne sais comment, il ne s'agit pas de cela, par rapport à moi qui fus ailleurs, qui serai ailleurs, et à ces endroits où je fus, où je serai » (24-25). Tout dans ce préambule laisserait attendre un récit de type personnel, n'étaient les réticences et leur redite insistante : "Je vais avoir à parler de choses dont je ne peux parler (...) Cependant je suis obligé de parler » (8). Ces réticences en effet renvoient déjà, par l'emboîtement des verbes de parole, à un autre projet qui n'est plus celui de raconter une histoire, mais celui de parler de ce dire qu'est parler. Et par le jeu des verbes modaux - devoir/ne pas pouvoir - elles renvoient aussi à l'enfermement de la parole dans un conflit qui la mine. Ecouter le parleur de L'Innommable qui dans sa "rage de dire » est en quête des moyens de "poursuivre » le discours puisqu' «il

${ }^{3}$ G. GENETTE, Nouveau discours du récit, Seuil, 1983, p. 35, et D. COHN, La Transparence intérieure, Seuil, 1981, p. 245. 
faut que le discours se fasse " (12), c'est donc partir à la rencontre d'un texte multiple, distendu entre des projets contraires - parler de soi mais aussi parler de l'impossibilité de parler - et hanté par le paradoxe d'un échec, ne pas pouvoir parler, que contredit violemment le fait de parler.

$\mathrm{Si}$ donc le récit vient prendre place dans ce contexte, c'est en se construisant selon le principe d'une convocationrévocation dont l'incipit a fermement tracé le programme : "Dire je. Sans le penser » (7). "Cela, dire cela, sans savoir quoi » (7). " Gens avec choses, gens sans choses, choses sans gens, peu importe, je compte bien pouvoir balayer tout ça en très peu de temps » (9). On sait d'ailleurs, et Ricardou l'a clairement rappelé 4 , qu'on ne saurait, dans l'ordre textuel, détruire le récit sans le prendre pour tremplin de la destruction, tant il est vrai que dénoncer, c'est encore énoncer pour montrer qu'on renonce. Suivre au fil du texte l'activité subversive d'un locuteur qui conduit le procès de son récit selon une stratégie très consciente et très calculée pour retrouver derrière ce parleur un Beckett attaché à déstabiliser le socle des idées sur lesquelles s'est érigée, avec la pensée subjectiviste, toute une littérature romanesque imprégnée d'humanisme est une entreprise fascinante que la critique beckettienne a conduite avec passion. Notre propos, beaucoup plus limité, sera ici de lier cette subversion à un problème qui est celui de la voix narrative.

«Curieuse tâche, que d'avoir à parler de soi » (40)

Premier pas dans la désagrégation du récit, le coup porté au narrateur par le brouillage de son identité. Dès le départ, tournant autour d'un "je » diégétisé, une histoire s'amorce qui déjà se délite, tissée de questions et d'hypothèses avant d'être brutalement congédiée : "Mensonges que tout cela " (29). Un peu plus loin cependant : "Décidément, se dit le parleur, je vais me prêter encore un peu à cette histoire, il n'est pas impossible qu'il y ait du véridique là-dedans » (57). Mais la source de l'histoire est alors attribuée, non sans circonlo-

4 J. RICARdoU, Le Nouveau Roman, Seuil, 1973: «Avec le Nouveau Roman, le récit est en procès : il subit à la fois une mise en marche, et une mise en cause ». 
cutions, à un certain Mahood (37). Et raconter, c'est alors " réciter ", ce que fait le parleur avant de déléguer son rôle à un certain Worm. "Ici c'est la voix de Worm qui commence (...) A Worm de jouer, on lui passe la main, je lui souhaite bien du plaisir » (98-99). Or c'est pour déclarer mais bien plus tard qu'après " avoir tâté de Mahood et autres Worm, maintenant c'est moi qui dégoise » (176) et pour reconnaître enfin que Mahood, «C'est moi qui l'ai inventé, lui et tant d'autres, et les endroits où ils passaient, les endroits où ils restaient, afin de pouvoir parler, puisqu'il fallait parler, sans parler de moi » (182).

On ne saurait plus efficacement saper le pouvoir de " l'instance narratoriale » que de la soumettre ainsi à manipulation pour la réduire au rôle de masque ou de prête-nom et la renvoyer à tous les aleas de la nomination: "Tiens, c'est peut-être en voulant être Worm que je serai enfin Mahood. Alors je n'aurai plus qu'à être Worm. Ce à quoi je parviendrai sans doute en m'efforçant d'être Tartempion. Alors je n'aurai plus qu'à être Tartempion. Halte-là (...) Worm, Worm, à nous trois, et vogue la galère » (88). Vogue la galère du récit sous l'égide de ce narrateur qui, de simulation en dissimulation, n'est plus, dans l'indétermination de son identité, qu'un " préposé » (196), à recentrer tardivement, et par effet de dilation, sur le pronom je, un pronom qui, dans cette histoire de soi par soi, est à la fois source et objet de la parole.

\section{«Je suis celui qu'on n'aura pas » (87)}

Or ce je va lui aussi se révéler insaisissable, parce qu'il est pris dans les ambiguïtés de la narration, cette narration où chacun des supposés narrateurs se voit projeté comme personnage sous la gouverne d'un même je et sous l'identité d'un même moi. Plus rien ne permet de distinguer le nommé Mahood du nommé Worm, du non-nommé je, tous devenus interchangeables dans le récit comme le souligne le parleur lorsqu'il se donne pour maître du jeu : " Il va falloir que je lui donne un nom, à ce solitaire. Sans noms propres, pas de salut. Je l'appellerai donc Worm (...) Ce sera mon nom aussi, au moment voulu, quand je n'aurai plus à m'appeler Mahood (...) L'essentiel est que (...) je ne sois jamais nulle part, ni chez Mahood, ni chez Worm, ni chez moi, peu importe grâce à 
quelle dispense » (84-86). Et d'ajouter de façon plus abrupte, et plus décisive à coup sûr : "Je suis celui qu'on n'aura pas » (87).

On le voit, qu'il soit à l'origine de la parole ou qu'il s'inscrive dans l'histoire que porte cette parole, le pronom personnel n'est plus qu'un terme flottant, privé de toute consistance sémantique. Instable et pluralisé, valant pour n'importe quel nom de baptême, il est renvoyé à son statut grammatical de pro-nom, pure disponibilité au sein du logos qui l'ouvre à tous les aléas de la référenciation. Mais il est aussi et au même moment décentré, et la perte de sa position hiérarchique en fait le lieu où se joue la dissolution du sujet au sens philosophique du terme. Un "sujet » réduit à sa fonction syntaxique, néantisé comme conscience et comme pensée, et condamné au pensum d'aligner des mots vides de sens. «Croient-ils que c'est moi qui parle ? Ça, c'est d'eux aussi. Pour me faire croire que j'ai un moi à moi et que je peux en parler, comme eux du leur» (98) et plus tard «Je dis je en sachant que ce n'est pas moi » (197). N'émerge de ce pronom que le cri de la fin : " Me voilà l'absent, (...) celui qui ne parle ni n'écoute, (...) il n'a pas d'histoire, il n'a pas été dans l'histoire, (...) il est dans son histoire à lui, inimaginable, indicible » (210-11).

Qu'en est-il alors de cette histoire qui seule peut donner quelque crédit à l'existence d'un narrateur ? A la voir se construire par lambeaux car il y a l'histoire de Mahood, puis l'histoire de Worm, et, dans la dispersion de ces données, celle du parleur, on ne peut qu'être surpris de l'étrange similitude des récits successifs; tous viennent dire en effet, en s'enroulant l'un sur l'autre, le même enfermement et la même solitude, dans le gris ou le noir, le même corps douloureux, une tête aux yeux exorbités ou révulsés ou hagards, d'où ruissellent les larmes, un tronc mutilé, un visage qui n'est plus que le trou des orbites ou de la bouche. Histoire d'un délabrement, et récit lui-même délabré, enclavé dans une parole qui le déborde de toutes parts. Histoire piétinante, vouée au statisme que lui impose l'absence d'un schème cohérent: temps arrêté " je suis ici, (...) depuis toujours » (24-25), personnage immobile « je n'ai jamais été ailleurs qu'ici » (62), et parole ressassante, 
elle-même tentée par le surplace de la redite et le tournoiement des affirmations aussitôt infirmées.

«C'est la présentation qui compte » (145)

Que lire dans ce propos narratif qui se déroule sans progression de l'information, sans construction d'une intrigue, sans création psychique du personnage, sinon la présence sarcastique du meneur de jeu qui s'attache à dérégler le récit en le soumettant à un système de variation auquel n'échappe aucun des paramètres du modèle canonique.

Ainsi de la donnée spatiale qui se voit, comme toujours chez Beckett, richement modulée puisque ici, ce sera au fil du texte un endroit dans le noir, une cour entourée de hautes murailles, une jarre profonde dans une rue peu passante près des abattoirs, une enceinte soigneusement close, sorte de cachot, le tout associé à la formule « Je suis ici » qui ne varie pas.

Autre donnée élémentaire, le temps des verbes qui va brutalement déplacer le récit au présent et le construire à l'imparfait sans que s'esquisse le moindre repère chronologiques. $^{-}$.

Quant à la variable pronominale, elle ne pouvait qu'appeler la transformation du je en $i l$, si bien que le je parle devient un «celui qui parle" fortement modalisé par le dubitatif : «il a dû voyager, il a dû voir quelques hommes, quelques choses, il a dû être là-haut, sous la lumière, ou bien on lui a raconté des histoires » (194). Je, il, quelqu'un, on,

${ }^{5}$ La subversion des normes de l'organisation temporelle exigerait une étude approfondie. Outre l'effacement de la distinction entre passé et présent, atténué d'ailleurs par l'opposition thématique ne pas bouger/bouger, il faudrait étudier le glissement constant de l'indicatif au conditionnel, et l'assimilation réitérée du singulatif et du duratif. Il nous suffit ici de souligner le statisme du schème temporel dans une fable que Beckett inscrit sous le signe de "l'incertitude vraisemblable " (12). Quant à l'énallage pronominal, il s'opère dans les deux sens [je $\rightarrow$ il (114) et il $\rightarrow$ je (128)] si bien que l'histoire de Worm, imbriquée dans celle du locuteur, apparaît comme l'une de ses versions, la plus ironiquement distancée (107). 
« lui, moi, peu importe » (196), comme le dit le parleur dans le tournoiement douloureux d'un constat d'aporie.

Si l'on ajoute à ces repères grammaticaux, la donnée lexicale, on voit que tout du récit se laisse conduire par le principe d'un recours à l'antonymie : bouger/ne pas bouger ; parler/se taire ; être/n'être pas, mais d'une antonymie qui s'annule au fil du texte.

Le récit alors apparaît comme un récit paradoxal où, de version narrative en version narrative, les pronoms, les temps verbaux, les adverbes spatio-temporels, et les mots-thèmes eux-mêmes font l'objet d'une identification des contraires par le jeu d'une opération d'investissement sémantique où l'un vaut l'autre si bien que tout s'annule par résorption de la différence dans la similitude et de la pluralité dans l'unité.

Et s'il fallait douter de cette lecture où le récit en se désagrégeant s'exhibe comme artefact, les commentaires du narrateur dissiperaient bien vite toute incertitude sur ce point. Car toutes ses remarques concourent, en dénonçant l'arbitrarité de la fiction, à souligner le principe de cette annulation par mise en équivalence des contraires. Ainsi du pronom : "Bah, peu importe le pronom pourvu qu'on n'en soit pas dupe » (94) et, plus vigoureusement encore, " Je ne dirai plus moi, Je ne le dirai plus jamais, c'est trop bête, je mettrai à la place $(. .$.$) la troisième personne, si j'y pense (. .$.$) ça ne$ changera rien » (114). De même, pour le temps choisi : "Hé oui, tantôt c'est le passé, tantôt le présent (...) la portée des termes hier et aujourd'hui m'échappe plutôt » (68-70). Quant à la variation à laquelle sont soumises les données narratives, elle suscite le sarcasme : "Cette version des événements rétablie, il ne reste plus qu'à remarquer qu'elle ne vaut pas plus cher que l'autre » (61). D'ailleurs, «il n'y a plus rien, il n'y a jamais eu rien, à tirer de ces histoires » (154). Et l'implosion du récit sera sanctionnée par le geste verbal d'un effacement plus radical « qui est-ce qui parle, ce n'est pas moi qui parle... ce sont les autres qui parlent... je les entends, je suis muet » (165) et même plus loin « je n'ai jamais parlé. J'ai l'air de parler » (195).

Le bilan s'impose, une histoire est là, mais c'est une histoire "vaine » (47) et son narrateur n'a de voix que pour en souligner l'inanité, au moment où il la parasite et l'obstrue 
de ses remarques critiques. Narrateur épisodique de surcroît, et prompt à se poser en fabulateur qui efface une erreur par une autre erreur (66) et qui, d'annulation en annulation, devient le fossoyeur de son propre récit.

C'est en surplomb de ce narrateur qu'il faut situer le seul point fixe du texte. Quelqu'un est là qui parle et se parle et ne cesse de se parler, affirmant qu'il invente (43), qu'il se trompe, s'est trompé, se trompera encore $(20,28)$, que ce qu'il ne dit pas, il le dira peut-être (65) et qu'au total il ne sait pas ce qu'il dit $(81,86)$. Peu importe d'ailleurs puisque « ce qui se passe, ce sont des mots", que toute cette histoire, c'est "une histoire de mots à dire » (45) et qu'on ne dit jamais qu'une seule chose, la même que toujours (81), à savoir qu'on est «à tout jamais personne » (87). Aussi n'y a-t-il « qu'à errer et à laisser errer, de mot en mot, qu'à être ce lent tourbillon sans bornes (...) Quelqu'un parle, quelqu'un entend, pas besoin d'aller plus loin, ce n'est pas lui, c'est moi, ou un autre, ou d'autres, qu'est-ce que ça peut faire » (192).

Or dans cette parole qui se refuse à coïncider avec le récit, parole sans frontières que hante la seule question qui vaille «Je. Qui ça ? » (83), ce qui s'impose avec force, c'est la présence d'une voix, la voix d'un parleur anonyme, attentif à ces mots qu'il dit, ces mots qu' "il faut dire, tant qu'il y en a », «ce sont des mots, je n'ai que ça » (211-212), des mots qu'il scrute parce que c'est eux qui portent son histoire ; mais attentif aussi à la voix d'où viennent ces mots, cette voix «qui n'est pas la mienne, mais qui ne peut être que la mienne » (34). Ma voix, la voix, cette voix, sa voix «tissée dans la mienne » (38), terme obsédant pour le parleur que hante la nécessité d'une réflexion sur ce point: " C'est que c'est une question de mots, de voix, il ne faut pas l'oublier, il faut essayer de ne pas l'oublier complètement » (162).

\section{"Cette voix qui parle» (128)}

La voix qui parle. Là est dans L'Innommable la véritable présence, celle qui, en s'élevant sur les ruines du récit, porte le texte de bout en bout, et bien au-delà de ses enclaves narratives, au point d'en constituer le foyer dynamique, au point surtout de donner corps à l'un des thèmes majeurs du livre. 
Foyer dynamique, à coup sûr : le dramaturge qu'est Beckett sait, projetant dans l'écrit tous les pouvoirs de l'oralité, assurer la mise en scène de la voix. Voix multiple du parleur, porteur d'ironie, de sarcasmes, de gravité ou d'angoisse, de provocations et de dérision; de détresse et de violence toujours. Et, dans cette " écriture à haute voix » 6 , la richesse de l'inventivité verbale est telle que, par le dialogue des postures énonciatives, le texte se fait représentation, au sens théâtral du terme : représentation ou jeu de rôles, un jeu complexe et subtil entre locution et métalocution, narration et métanarration, voix en acte du parleur et verbalisation de la mise en scène de cette voix. Et comme le Beckett posté derrière son parleur devance toujours son lecteur, on ne s'étonnera pas d'entendre ce locuteur se présenter comme " le même sale individu s'amusant à paraître multiple, en changeant de registre, d'accent, de ton, de bêtise ", non sans ajouter aussitôt « A moins qu'il ne soit réellement ainsi » (108). Car, le texte le souligne, la voix dans la richesse de ses inflexions peut se donner en "spectacle » (158); alors elle joue, qu'elle récite ou qu'elle improvise, mais elle peut aussi, jetant le masque du comédien, se faire l'interprète des contradictions de l'être, exprimer notamment les déchirements du moi, ce «moi qui hurle, loin derrière ma dissertation » (46).

Il y a plus : d'être présence vive, la voix n'en est pas moins aussi objet de réflexion. Evoquée tantôt comme l'organe de la parole, venue du corps, production sonore qui rompt le silence et qui s'entend, et qui "s'altère ", et "murmure " ou " vocifère ", tantôt comme l'expression du moi, marque identitaire, et cette voix-là, il faudrait la taire, ou

${ }^{6}$ La formule est empruntée, on le sait, à R. Barthes, Plaisir du texte, Seuil, 1973, p. 104. Métaphore, bien sûr, pour désigner dans l'écrit la simulation, tonale et rythmique, de l'oralité. Sur cette voix "en représentation ", voir aussi Y. Delègue, "La littérature-ventriloque ", Poétique 72, qui élargit la réflexion au problème de la mimésis et de l'identité du sujet.

Que cette présence vive de la voix crée dans le soliloque un espace de dialogue, tout le montre dans l'Innommable. Encore faut-il sous le mot dialogue distinguer ce qui est clivage des rôles assumés par le locuteur, ce qui est interpellation de l'autre par convocation d'un ils (141) ou d'un vous (112), et ce qui relève de l'autodérision. Autre sujet d'étude. 
bien alors la destituer de son pouvoir en la prêtant à d'autres. Evoquée surtout comme la compagne obligée de la pensée, inséparable de la quête de signification, et peu importe alors que la voix soit sonore ou muette, puisque c'est toujours dans les mots qu'elle se glisse : "Cette voix qui parle, se sachant mensongère, indifférente à ce qu'elle dit, trop vieille peut-être et trop humiliée pour pouvoir jamais dire enfin les mots qui la fassent cesser (...) Elle sort de moi, elle me remplit, elle clame contre mes murs (...) je ne peux pas l'arrêter, je ne peux pas l'empêcher, de me déchirer, de me secouer, de m'assiéger " (34). " Si cette voix pouvait s'arrêter, seulement une seconde, elle me semblerait longue, une seconde de silence » (128).

Voix de la pensée lancée en direction de ce qui se dérobe, et c'est cette pensée, tâtonnante, dans le noir, que dira la personnification des dernières pages "Une voix pareille, qui peut la contrôler, elle essaie tout, elle est aveugle, elle me cherche, dans le noir, elle cherche une bouche, où se mettre (...) elle est la seule, il faudrait une tête, il faudrait des choses, je ne sais pas, j'ai trop l'air de savoir, c'est la voix qui fait ça (...) la voix me dira tout, ce dont j'ai besoin, elle me l'a déjà dit, elle me le redira, tout ce dont j'ai besoin, par petites bribes » (207). Tourment et tournoiement de la pensée qui cherche à donner sens au monde et à la vie, et à « celui hors la vie qu'à la longue la longue vie vaine veut qu'on n'ait cessé d'être » (100), en se frayant un chemin, à l'aveugle, dans la précarité du langage.

Quel que soit en effet le point d'ancrage de la lecture, et qu'on regarde du côté du récit ou du côté du discours de ce parleur fasciné par le mot « voix », et par les jeux de la voix, on ne quitte jamais le domaine d'une réflexion sur l'univers des mots. Par la fiction d'un locuteur auquel il confie le soin d'élaborer sur le mode de la fable une histoire qui se délite et s'exténue, tour à tour parodique et dérisoire ${ }^{7}$, Beckett a inscrit le procès du récit dans un procès beaucoup plus radical, celui

${ }^{7}$ Parodiant tour à tour le récit de voyage, le récit d'apprentissage, et le récit d'amour et de vie et de mort, Beckett ironise sur l'effet " veillée des chaumières "jouer sur l'expression " on nous a raconté des histoires " $(53,147)$. Et dans l'insistance de ce refus du leurre narratif, la conscience aiguë du mensonge qu'est raconter. 
du langage, de son in-signifiance, et du vide que portent les mots, usés ou vains et mensongers.

«Toute cette histoire de voix est à revoir, à corriger, à démentir» (82)

Alors, dans ce texte si peu narratif et si violemment dénonciateur de son médium, est-il encore possible de parler de voix narrative?

De voix, certes, s'il s'agit par là de caractériser "l'instance productrice du discours ». Mais telle est cette voix, telle l'ironie de son propos qui affirme ne glisser vers «le secours de la fable » que pour se prolonger sans « tarir » $(36$, $35)$, telle aussi sa rébellion contre le vieux monde des conventions littéraires, que le discours qu'elle tient, en une errance «vaguement circulaire » (59), se place résolument sous le signe de l'in-quiétude et de la défiance : opaques ou trompeurs sont les mots, vaines les histoires, impasse le raisonnement ${ }^{8}$, imposture la logique du distinguo qui voudrait que oui soit le contraire de non et qu'on ne puisse parler si on est muet ou «notoirement aphone » $(34,147)$.

$\mathrm{Au}$ détenteur de ce discours, parleur lucide et lucidement conscient des ambiguïtés, des malentendus et des contradictions ou des illogismes de la parole, quel statut conférer en termes de narratologie?

Le désigner comme le narrateur, ce qu'il n'est que sporadiquement dans le texte, ce serait trahir une posture d'ensemble qui désoriente les schèmes ordonnateurs du récit et tout en les exhibant pour mieux les subvertir, prend appui sur leur délabrement pour montrer que parler, c'est se payer de mots face à un réel inaccessible (141).

${ }^{8}$ Les impasses du raisonnement sont tournées en dérision bien des fois dans l'Innommable et notamment p. 168 et 187. Dans ses entretiens avec Juliet (Ch. Juliet, Rencontres avec Beckett, POL, 1999) Beckett évoque d'ailleurs en ces termes l'illogisme des mystiques: «J'aime... leur illogisme brûlant... cette flamme... qui consume cette saloperie de logique » (p. 72). 
Songer à lui comme à un personnage, et sauf à rendre à ce terme la valeur de son étymon ${ }^{9}$, ce serait ignorer le refus plusieurs fois proclamé d' « entériner un vieil état de fait » et son incompréhensible "charabia » $(7,63)$, et méconnaître la volonté vigoureusement affirmée de donner au « moi », ou à la "personne », ou au " sujet », la seule définition possible, par le vide d'un terme sémantiquement silencieux ou par la négation de toute forme de positivation ${ }^{10}$.

Trouver refuge dans la notion d'instance énonciative supposerait qu'on clarifie un concept qui dans son globalisme fédérateur, désigne tout à la fois l'origine de la parole, les données situationnelles de cette parole et l'acte de communication" ${ }^{\prime}$. Un tel concept, d'ailleurs, s'appliquerait bien mal à un locuteur sans carte d'identité ni domicile fixe, et de surcroît porteur d'un discours cryptique.

Mieux vaut faire retour vers l'auteur dont la figure dans le texte implicitement se dessine. Et la densité dans L'Innommable du réseau des indices révélateurs de la présence beckettienne ne peut, de l'ironie au jeu généralisé sur la polysémie, et de la théâtralisation de la parole à la projection dans le récit des "créatures » de l'œuvre antérieure ${ }^{12}$, que conforter ce point de vue, dût-il conduire à

9 Par le mot persona, le latin désignait le masque de l'acteur, évoquant ainsi le dédoublement du jeu de rôle, alors qu'en grec, la langue convoyait, avec la notion d'hypocritès, l'idée de feinte et de latence.

${ }^{10}$ Beckett à Juliet : "La négation n'est pas possible. Pas plus que l'affirmation. Il est absurde de dire que c'est absurde. C'est encore porter un jugement de valeur. On ne peut pas postuler, et on ne peut pas opiner» (op. cité, p. 68). Une seule attitude possible face à "l'inintelligible": le "subir» (p. 54). Et pour l'exprimer verbalement, abolir toute distinction entre « je parle » et « je ne parle pas » (209); « je suis » et " je ne suis pas »; «Il n'y a que moi, moi qui ne suis pas, là où je suis " (114).

11 Sur ce globalisme du terme énonciation, voir dans C. KerbratOrecchioni, L'énonciation. De la subjectivité dans le langage, Colin, 1980, 28-33, l'analyse des glissements sémantiques qui se sont opérés.

12 Parmi ces «créatures » qu'il baptise aussi ses « délégués » (22, 17), Beckett convoque d'abord Malone, Molloy et Murphy, puis "le pseudo couple Mercier-Camier ", tous projetés dans l'histoire de son parleur où ils passent et tournent avant d'être brusquement congédiés 
repenser, dans son rapport au discours, les notions de récit, et de fabulation ${ }^{13}$.

Privilège de la fiction romanesque : en déléguant à un locuteur insituable dans le temps ou l'espace, et non identifiable, le soin de le représenter, l'auteur s'absente, mais pour s'avancer masqué, absent toujours et cependant présent. Figure du double, ou de l'ombre portée (9), face à laquelle le problème n'est pas de distinguer le faux du vrai mais le latent du manifeste, afin de mieux entendre, capté par la fascination du simulacre, les grandes interrogations d'une écriture obsédée par l'exigence de rompre avec le passé, et désormais travaillée par le doute à l'égard de ses propres pouvoirs.

par le narrateur: "Ils m'ont fait perdre mon temps (...) Que maintenant ils s'en aillent " (28). Au-delà, tous ces noms seront à nouveau fugitivement rappelés avec ceux de Watt et Moran, et toujours comme supports d'une négation de l'identité ou de la parole (65, 195) : autant de "pantins » (8) pour le fabulateur.

13 Au Récit la chronométrie inhérente à tout développement verbal, de quelque genre qu'il relève. A la Fabulation la part de duplicité et de dissimulation ou de non-dit de la parole sous ce qu'en manifeste le dit. 